\title{
Study of the surface size defects in the case of a soda lime glass eroded by sandblasting
}

\author{
A. Faci ${ }^{\mathrm{a}}$, M. Kolli, N. Bouaouadja and B. Chabane \\ Laboratory of Non-Metallic Materials, I.O.P.M., F. Abbas University, Sétif 19000, Algeria
}

\section{INTRODUCTION}

In Saharan regions, the sandstorm has an negative influence not only on the rural and urban environment, but also on the erosion of brittle materials, particularly glass products (home windows' glass, windshields and headlights of vehicles, solar panels protective glass...), or coated glass with thin layers (functional glasses). This influence often results in a deterioration of the surface by sand particles impacts. In fact, particles impacts on the glass surface generate various superficial defects (microcracks, craters and scaling) that scatter the incident light, and thereby reduces the optical transmission. Furthermore, the impact points of the damaged glass are privileged sites of sand dust deposits that affect in particular the photovoltaic efficiency of solar panels, visibility through windshields of vehicles [1]... According to literature $[2,3]$, the erosion process in glasses is considered as elastic-plastic. Imprints formed at the impact sites are accompanied by a network of cracks caused by the impact force. This results in a gradual weight loss during erosion. In the case of eroding sharp particles, there is a formation and growth of radial cracks on the surface. Lateral cracks that form at the base of the contact area converge to the outside almost parallely to the surface. In general, lateral cracks are responsible for the mass loss by scaling mechanism, while radial cracks are the cause of the mechanical resistance deterioration [4]. Hutchings [5] and other authors have reported that the erosion of brittle materials is affected by many factors:

- Properties of the incident particles (size, shape, density, hardness, fracture toughness);

- Properties of target materials (hardness, fracture toughness and surface state),

- Test conditions (impact velocity, impact angle and temperature).

In this work, the influence of two test parameters (flux velocity and impact angle) on the evolution of the surface defects generated by sandblasting is presented. A statistical study is applied on the obtained defects sizes distribution.

\section{EXPERIMENTAL PROCEDURE}

the used sand comes from Ain Amenas (Southern Algeria). Microscopic observations show that sand grains are of yellow brown color. They have a roughly round shape with a few angular grains. To facilitate this study, large particle

a e-mail: faciaziz@yahoo.com

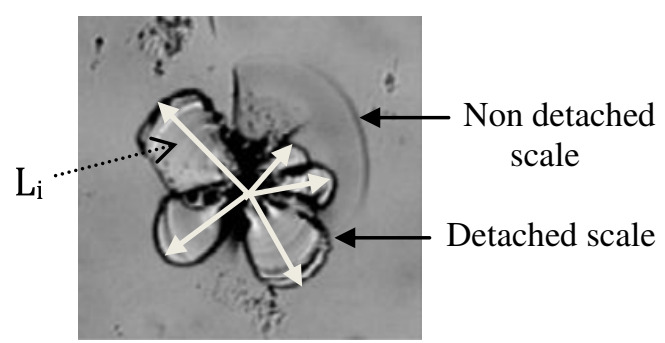

Figure 1. Typical defect and the scales length Li.

sizes enough $(1000 \pm 114) \mu \mathrm{m}$ and sand mass low enough $(30 \mathrm{~g})$ were deliberately chosen. This enables to obtain isolated impacts with well-defined scales. Also this choice allows avoiding interactions between particles themselves and between defects.

The studied material is soda-lime glass provided by the company Africaver (jijel, Algeria). Square samples of $50 \times 50 \times 3 \mathrm{~mm}^{3}$ dimensions were prepared from the same sheet. The samples are tested in their initial state, without previous treatments. They are eroded on the same side. Microscopic observations showed that the defects are of variable sizes and randomly distributed on the surface. Only defects of size higher than $15 \mu \mathrm{m}$ are considered. Beyond this size, the scaling shape becomes clearer and easy to measure. For the defects sizes measurements, we adopted Evans's method [2]. This involves measuring the size scales in the longitudinal directions (Figure 1). For each impact, we take the average length of detached scales Li. For the statistical study of defects, we randomly selected 50 individual impacts in an eroded surface of $1 \mathrm{~cm}^{2}$ in the central zone (most degraded) for each sample.

\section{RESULTS}

In this study, the variable parameters are: flux velocity $(15,25$ and $35 \mathrm{~m} / \mathrm{s})$ and impact angles $(30,50,70$ and $\left.90^{\circ}\right)$, while the constant parameters are sand masse $(30 \mathrm{~g})$ and sand size $(1000 \mu \mathrm{m})$. The results show that for the same flux velocity, the average size of defects increases as the incident angle increases and tends towards $90^{\circ}$. The effect of the particles kinetic energy is variable according to the impact angle value. In a normal position $\left(90^{\circ}\right)$, almost all of the kinetic energy is absorbed by the sample surface at the impact site level. Therefore, the size of defects is maximal in term of extent and depth. When the samples positions tend to be inclined, the tangential force component predominates, and consequently the defects 

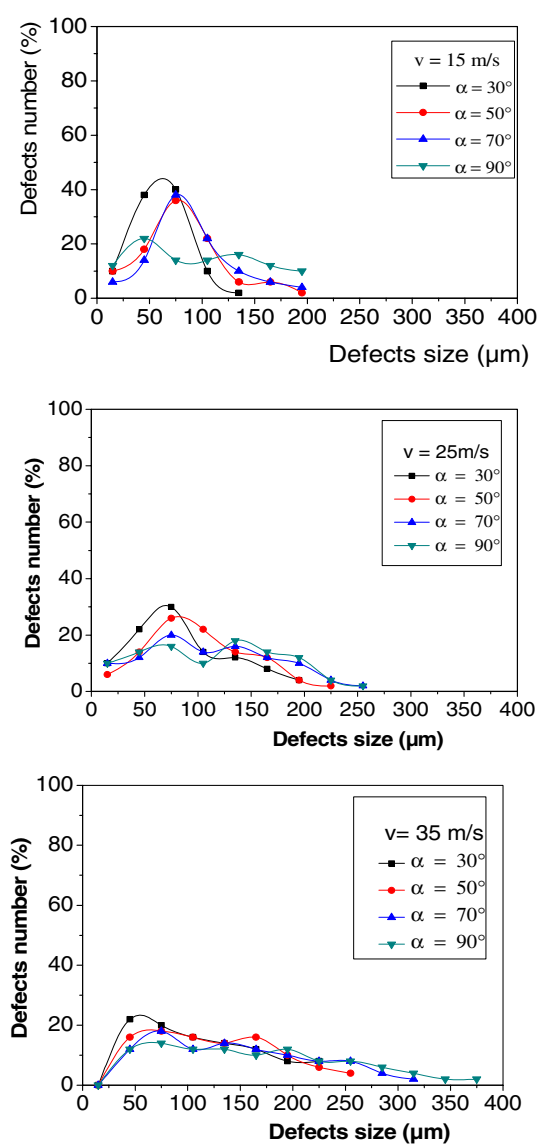

Figure 2. Variation of the defects number versus defects size for $\mathrm{V}=(15,25,35 \mathrm{~m} / \mathrm{s})$ and different angles.

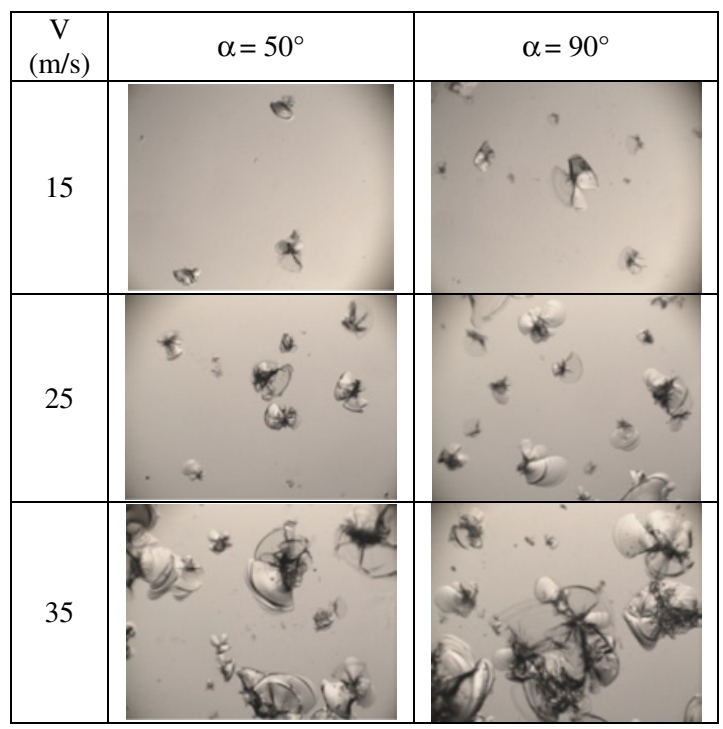

Figure 3. Some micrographs of the surface defects obtained for two impact angles $\left(50\right.$ and $\left.90^{\circ}\right)$ and for various flux velocities.

size decreases. On another hand, the defects size and their numbers reach their minimal value as the impact angle approaches $30^{\circ}$. As example for a constant flux velocity $(\mathrm{V}=15 \mathrm{~m} / \mathrm{s})$, the maximum defects size passes from $128 \mu \mathrm{m}$ for an angle of $30^{\circ}$ to $195 \mu \mathrm{m}$ for $90^{\circ}$ angle. The maximum defects number is about $40 \%$ (Figure 2).
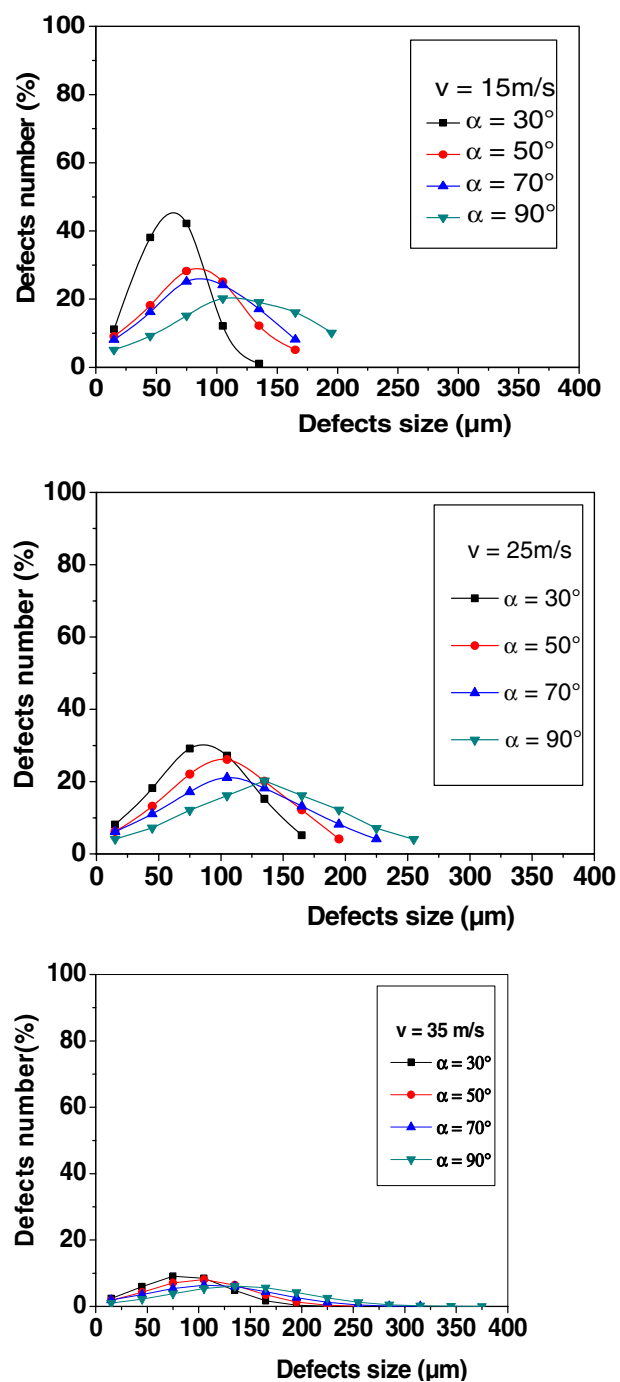

Figure 4. Variations of the defects number versus their sizes for various flux velocities $(15,25$ and $35 \mathrm{~m} / \mathrm{s})$ and impact angles (30, 50,70 and $90^{\circ}$ ).

When the velocity of the sand flux increases from 15 to $35 \mathrm{~m} / \mathrm{s}$, the surface damage also increases, by formation of defects with various sizes. This is the result of the particles kinetic energy which increases. So, we can assume that the only relevant parameter is the velocity of impact, because the sand mass is kept constant. Similarly, we can see that as the speed increases from 15 to $35 \mathrm{~m} / \mathrm{s}$, the defects number $\mathrm{N}$ falls steadily $(40,35,22 \%)$, while the average defects size D is steadily increasing $(195,255$, 375 microns). For example, for $\mathrm{v}=15 \mathrm{~m} / \mathrm{s}, \mathrm{N}=40 \%$ and $\mathrm{D}=225$ microns, while for $\mathrm{v}=35 \mathrm{~m} / \mathrm{s}, \mathrm{N}=30 \%$, and $\mathrm{D}=369$ microns

It can be concluded that when the velocity increases, the defects size increases while the maximum defects number decreases. For the defects of large sizes obtained for $\mathrm{V}=35 \mathrm{~m} / \mathrm{s}$, the defects number distribution as a function of their sizes is almost uniform, and regularly decreases from a size of $190 \mu \mathrm{m}$. In other words, all the defects sizes tend to have almost the same number regardless of the impact angle. This trend can be explained by the dominant effect of the flux velocity on the 
distribution of the number of defects according to their sizes. For sand grains of constant size, as in our case $(1000 \mu \mathrm{m})$, the defects size increases with increasing the impact velocity. This is linked to the increasing of the kinetic energy of incident particles. In general, the defects size increases when the impact angle tends towards $90^{\circ}$.

Figure 3 shows some micrographs of the defects obtained for two impact angles (50 and $90^{\circ}$ ) and for various flux velocities. It appears clearly that for each impact angle when the velocity of particles increases, the defects number and their size increase. As consequence, the possibility of interaction between neighboring defects becomes easier. Similarly, when the impact angle approaches $90^{\circ}$, the damage increases and tends to spread over the entire exposed surface, especially if the impact speed increases. This behavior is related to the kinetic energy of the incident sand particles which increases. Through these micrographs, we can also confirm the dominant character of the velocity relative to the impact angle because the damaged surface increases more rapidly with speed than with impact angle.

Figure 4 shows the variation of the defects number versus their sizes according to the normal law. It appears clear that the curves for the three flux velocities present a positive dissymmetry. This means that the major part of surface defects presents a small size. The maximum defects size for angles 70 and $90^{\circ}$ reaches $375 \mu \mathrm{m}$. For the highest speed $35 \mathrm{~m} / \mathrm{s}$ the curves are nearly similar and very flat and the defects number decreases strongly
$(<6 \%)$. This confirms the dominant character of the flux velocity effect on the defects size distribution compared to the impact angle effect. The prevailing effect of the impact flux velocity on defects size was shown previously.

According to the obtained results, we note that:

- For lowest speed $(15 \mathrm{~m} / \mathrm{s})$, there is no independence between the incidence angle and the defects number per size.

- For highest speeds (25 and $35 \mathrm{~m} / \mathrm{s}$ ) there is independence between the two variables: the incidence angle and the number of defects.

\section{References}

[1] N. Bouaouadja et al., Etude des possibilités d'amélioration de la transmission optique d'un verre sodocalcique érodé par sablage. Verre, Céramique et Composites, 1 (2011) 43.

[2] A. G. Evans, M. E. Gulden, M. Rosenblatt, Impact damage in brittle materials in the elastic-plastic response regime. Proc. R. Soc. London, Ser. A, 361 (1978) 343.

[3] S. M. Wiederhorn, B. J. Hockey, Effect of material parameters on the erosion resistance of brittle materials. J. Mat. Sci, 18 (1983) 766.

[4] J. E. Ritter, P. Strzepa, K. Jakus, L. Rosenfeld, K. J. Buckman, Erosion damage in glass and alumina. J. Am. Ceram. Soc., 67 (1984) 769.

[5] I. M. Hutchings, Tribology. Edition Edward Arnold (1992). 